\title{
ENERGÍA RENOVABLE A PARTIR DE LA BIOMASA DE LA CAÑA DE AZÚCAR
}

\author{
RENEWABLE ENERGY FROM SUGAR CANA BIOMASS
}

Leidy Verdezoto; Fernanda Parco; Carlos Jácome; William Katan; Alexandra Mora.

Universidad Estatal de Bolivar, Guaranda-Ecuador.

Email: leiverdezoto@mailes.ueb.edu.ec

https://doi.org/10.33789/talentos.8.1.140

\begin{abstract}
Resumen: El presente trabajo da a conocer los sistemas de cogeneración de energía a partir de la biomasa obtenida del bagazo de la caña de azúcar, aprovechando este residuo como combustible en los ingenios azucareros para alimentar calderas de alta presión, evitando costos de traslado del bagazo para su descomposición, obteniendo una fuente generadora de energía renovable. Un sistema de cogeneración es la generación secuencial o simultánea de múltiples formas de energía útil (generalmente mecánica, térmica y eléctrica) siendo $1 \mathrm{~kg}$ de bagazo igual a 37.5 MJ obteniendo por zafra 19,5 $\mathrm{t} \mathrm{ha}^{-1}$, en términos de energía a $13.86 \times 10^{5} \mathrm{kcal} \mathrm{t}^{-1}$. Este permite evitar pérdidas en forma de entalpía o exergía. La cogeneración es un instrumento clave para la eficiencia energética y la reducción de emisiones de GEI, evitando 7 millones de toneladas de $\mathrm{CO} 2$ al año. Los sistemas de cogeneración son eficientes energéticamente, brindan mayor competitividad al momento de generar energía, también permite exportar energía a otros sistemas si existe excedentes, lo que crea fuentes de empleo además de colaborar con el medio ambiente ya que se utiliza de una manera más eficiente los combustibles utilizados en el proceso.
\end{abstract}

Palabras Clave: caña de azúcar, cogeneración, desechos orgánicos, energía, proceso.

Abstract: This paper presents the energy cogeneration systems from biomass obtained from sugarcane bagasse, taking advantage of this residue as fuel in sugar mills to feed high pressure boilers, avoiding costs of transferring the bagasse for its decomposition, obtaining a generating source of renewable energy. A cogeneration system is the sequential or simultaneous generation

Recibido: 05 de enero de 2021

Aceptado: 11 de marzo de 2021

Publicado como artículo científico en la Revista de Investigación Talentos VIII (1), 9-26 
of multiple forms of useful energy (generally mechanical, thermal and electrical) with $1 \mathrm{~kg}$ of bagasse equal to 37.5 MJ obtaining per harvest $19.5 \mathrm{tha}-1$, in terms of energy at $13.86 \times 105$ $k c a l t-1$. This allows to avoid losses in the form of enthalpy or exergy. Cogeneration is a key instrument for energy efficiency and the reduction of GHG emissions, avoiding 7 million tons of CO2 per year. Cogeneration systems are energy efficient, they provide greater competitiveness when generating energy, it also allows energy to be exported to other systems if there is surplus, which creates sources of employment in addition to collaborating with the environment since it is used in a more efficient way the fuels used in the process.

Keywords: sugar cane, cogeneration, organic waste, energy, process.

\section{INTRODUCCIÓN}

Entre las biomasas producidas comercialmente, la caña de azúcar destaca, por su capacidad de fotosíntesis. La caña de azúcar crece en el trópico y en algunas regiones subtropicales, las características fisicoquímicas e investigaciones realizadas se han determinado, que el bagazo de caña es una fuente considerable de biomasa, la cual es aprovechable para la cogeneración de energía (Debernardi De La Vequia; Laurel Ortiz; Calleja Rosas, 2016).

A partir de la biomasa de la caña de azúcar y de otros compuestos orgánicos, esta metodología ayuda a reducir el consumo de combustibles de origen fósil generando procesos más eficientes, produciendo energía a base de productos renovables (Hernandez, Gonzalez, \& Freide, 2017).

Se considera como Biomasa a todos los desechos orgánicos, que se originan en la vida cotidiana del hombre, en los procesos industriales, en crianza de ganado, descomposición de basura, entre otros (Aguirre, 2015).

(Aroca, 2015) Si comparamos una central eléctrica convencional con una central de cogeneración, vemos que, en la central convencional, en el mejor de los casos, tiene una eficiencia del $60 \%$, es decir, que por cada 100 toneladas de carbón u otro combustible, se desperdician 40 toneladas, Por otro lado, una planta de cogeneración puede tener una eficiencia del $90 \%$ o incluso más. Esto supone un enorme ahorro de combustible y de costes de explotación, así como un gran beneficio medioambiental.

En México la biomasa generada por el cultivo de caña de azúcar (Saccharum spp) es un factor importante en términos de energía renovable: En la cosecha 2013-2014 se molieron 52,246508 $\mathrm{t}$ de caña en 52 ingenios azucareros, y la biomasa aprovechable energéticamente de la caña, es el bagazo y puntas de tallo, dándonos una fuente de energía de 57277234.6 TJ (Debernardi-De La Vequia H. e., 2016).

El Gobierno gallego ha desarrollado en los últimos años una normativa específica para el fomento de producción de energía eléctrica mediante el aprovechamiento de la biomasa 10 forestal primaria (Rodríguez \& Fernández, 
2014).

En Colombia, la cogeneración ha venido estimulándose desde la expedición de la Ley 788 de 2002, donde se crea una extensión a la renta generada por la venta de energía proveniente de biomasa, varios de los incentivos a la cogeneración contemplados en la legislación actual, se disipan por cuenta de las exigencias de la misma ley para hacerse acreedores a ellos. Sin embargo, el marco legal se ha ido ajustando el 16 de julio de 2008, entró en vigencia la Ley 1215, mediante la cual se exime a los cogeneradores de pagar la contribución del $20 \%$ sobre la energía que generen para su consumo. Posteriormente, a partir de diciembre de 2011 esta exención se extendería a todos los industriales que la soliciten (Corrales, 2017).

La cogeneración se ha identificado hace unos años atrás ya se han venido implementando, gracias a ello en Colombia en el año 2016 la capacidad instalada de cogeneración fue de 253 MW. La capacidad instalada de excedentes que hoy en día se venden es de 93,6 MW (Asocaña, 2017).

En ecuador existen estudios relevantes en el aprovechamiento de biomasa residual de banano que apuesta una alternativa sustentable y amigable para el medio ambiente. (Aguilar, 2019) Afirma que el potencial energético que podría obtenerse de 12849.57 T/año de biomasa residual de banano, representaría la cogeneración de 9.94Gwh/año de energía que podrá satisfacer la demanda energética en promedio de 1560 de familias.

En los últimos años una pequeña cantidad de energía renovable, proveniente de turbinas eólicas, paneles solares, plantas geotérmicas, biocombustibles, biomasa y otras, se han sumado al sistema de generación renovable, el cual se implementó en Estados Unidos (Rosero, Garcia, Minchala, Pozo, \& Morales, 2013). Según (Bohórquez, 2019) El potencial energético de los RSU de 28 a 44 MW eléctricos. Las estrategias termodinámicas para aprovechar energéticamente los RSU.

La caña de azúcar nos ofrece alimentos, combustible, energías renovables (Debernardi De La Vequia; Laurel Ortiz; Calleja Rosas, 2016). Es por ello que el objetivo de este trabajo es dar a conocer la utilidad del bagazo de caña de azúcar para la obtención simultánea de energía térmica en los procesos agro productivos.

\subsection{Caña De Azúcar}

Es el nombre común de esta especies de herbáceas, vivaces, de tallo leñoso de un género (Saccharum) de la familia de las gramíneas (Gramineae), originaria de la Melanesia y cuya especie fundamental es Saccharum officinarum. Fue introducida en Cuba por el año 1535 desde Santo Domingo. La caña de azúcar es cultiva en países tropicales y subtropicales, los tallos alcanzan entre 3 y $6 \mathrm{~m}$ de altura y entre 2 y $5 \mathrm{~cm}$ de diámetro (Casa, 2020). El sistema radicular lo compone un robusto rizoma subterráneo; el tallo acumula un jugo rico en sacarosa, compuesto que al ser extraído y cristalizado en el ingenio forma el azúcar. La sacarosa es sintetizada por la caña gracias a la energía tomada del sol durante la fotosíntesis con hojas que llegan a alcanzar de dos a cuatro metros de longitud. En su parte superior encontramos la panocha, que mide unos 30 
cm. de largo (Oliveira \& Pellegrini, 2010).

\subsection{La Caña De Azúcar Y La Cogeneración}

El bagazo es quemado en calderas para producir vapor y después conducido a una turbina donde se expande y da lugar a la producción de energía mecánica la cual es empleada para mover un generador eléctrico (Marroquin, 2015).

La generación de vapor opera a 43 atm y mayor eficiencia (85\%). Los turbogeneradores de extracción, condensación, puede operar a una presión de $43 \mathrm{~atm}$ y temperatura 406 ${ }^{\circ} \mathrm{C}$ (Chiriboga, 2018). El condensador tiene mayor flexibilidad, siendo posible operar durante todo el año. Con el bagazo disponible, se genera el vapor demandado por el proceso tecnológico y con el exceso se genera electricidad. La presión de condensación es de 0,085 atm (Abreu, González, Rico, Morales, \& Espinosa, 2016).

El total de producción de toneladas de bagazo diario, el ingenio azucarero aprovecha el $90 \%$ para la combustión directa en la planta de cogeneración, que transforma la energía térmica a eléctrica con una eficiencia del $40 \%$, además se estima dos semanas al año para el mantenimiento, por lo que la planta opera alrededor de 355 días (Castro, 2017). Toda esta biomasa que es quemada podría ser aprovechada como una alternativa renovable de producción de energía, diversificando así la matriz energética (Blanco \& Arce, 2014).

La caña de azúcar es uno de los cultivos con mayor capacidad de convertir la energía solar en biomasa (José Serrano W. M., 2017). Si se toma en cuenta solo el bagazo y la paja, en los cañaverales se almacenan alrededor del equivalente a una tonelada de petróleo por cada tonelada de azúcar (EspinosaMancinas, 2017). Según (Sardiñas, 2017) La combustión de la biomasa no incrementa la concentración atmosférica de carbono, porque solo devuelve a la atmosfera el carbono que fijo la planta durante su crecimiento. La biomasa aprovechable energéticamente son el bagazo y los RAC. El bagazo representa el $30 \%$ de los tallos verdes molidos y es el residuo fibroso de la molienda; se obtiene con un $50 \%$ de humedad, esto significa que por cada hectárea cosechada es posible obtener anualmente 13,5 ton de bagazo equivalentes a 2,7tce (tonelada de combustible equivalente: 37.5 MJ/Kg) (Debernardi-De La Vequia \& Rosas-Calleja, 2016).

\subsection{La Caña De Azúcar Como Fuente De Energía Renovable En América Latina}

América Latina presenta un gran potencial para la producción de caña de azúcar. Brasil, mayor productor mundial de caña de azúcar, en los últimos años ha contribuido con aproximadamente 800 millones de toneladas anuales (José Serrano W. M., 2017). Según (Fábio Ricardo Marín, 2018) El segundo mayor productor de caña de azúcar en América Latina es México, seguido por Colombia, Guatemala y Argentina. El área cultivada en Brasil, es destina aproximadamente 3,5\% de su área agrícola para ese cultivo (Sousa, 2018).

La caña de azúcar representa alrededor del $65 \%$ de la producción mundial de este alimento, siendo América Latina una de las mayores productoras, correspondiéndole el $37 \%$ del azúcar y el 58\% del total mundial de caña producida. (Paz-Enrique, Hernández- 
Alfonso, \& Peralta-González, 2016) La cosecha, en América Latina, es de 450 millones de toneladas de caña - 320 para la producción de azúcar y 130 para la producción de alcohol directo del jugo, inducen una serie de residuos y residuales, que son o que pueden ser, fuentes de combustibles y energía.

Esta producción se realiza con un combustible renovable y no contaminante que no es necesario transportar a las fábricas, pues es parte componente de la materia prima (Alexis Alonso-Gómez, 2018). La producción del azúcar crudo se puede realizar sin el uso de combustible o energía adicional a la que puede ser producida por el bagazo, existiendo un consumo de energía mecánica del orden de los 25-30 kWh/ton caña y un consumo de vapor para el proceso de unos $450-550 \mathrm{~kg}$ / ton caña. El bagazo, los residuos agrícolas de la cosecha (RAC) y la producción de biogás a partir de los residuales líquidos azucareros y alcoholeros son fuentes de combustibles y energía posibles de obtener de esta biomasa. (Valdes, Lopez, \& Isaac, 2017).

Hay muchos países como Bolivia, Costa Rica, Cuba, Colombia, Nicaragua, Honduras, Guatemala y El Salvador que consiguieron suplir por lo menos $20 \%$ de la demanda de energía eléctrica residencial con este sistema (Rosero \& L. Garza, 2016). Todos los indicadores muestran que muy pronto el $100 \%$ de la demanda residencial será atendida y por lo tanto, habrá electricidad excedente para satisfacer otros sectores. Considerando $0,5 \%$ del área de pastaje, el bagazo excedente sería suficiente para suplir 50\% de la demanda de electricidad en casi todos los países, excepto Jamaica, República Dominicana,
Ecuador, Perú y Venezuela (Claudio Carpio, 2013). En particular, Guatemala, Bolivia, Argentina y Paraguay podrían sustituir 100\% de la demanda de electricidad residencial y aún generar excedente para otros sectores. En general, todos los países podrían tener significativas contribuciones en la matriz eléctrica derivadas del bagazo (Benitez, 2016).

\subsection{Cultivos De Caña De Azúcar En Ecuador}

En Ecuador se estima que existen 110.000 hectáreas de cultivos de caña de azúcar, 74.100 están destinadas a la producción de Azúcar y el resto para la producción de panela (Largo, 2015). Se debe señalar que existen dos periodos en los ingenios azucareros, de enero a junio se realiza un mantenimiento preventivo a todas las instalaciones, debido que el invierno no permite ingresar de la manera adecuada a los cañaverales, el segundo periodo es conocido como zafra, que va desde de julio a diciembre donde se realiza la cosecha del cultivo, elaboración de azúcar y derivados en las centrales azucareras (Tigua, 2017).

Las principales zonas predominantes en cultivo de caña de azúcar son: Cotopaxi, Chimborazo, Los Ríos, Guayas, Azuay, Loja, Pichincha, Imbabura y Pastaza, donde el 92\% de la producción de caña se da en la Cuenca Baja del río Guayas (Pozo \& Suárez, 2018). En nuestro país existe varios tipos de caña cultivadas, estas variedades se han desarrollado en laboratorios de los ingenios azucareros, con el apoyo de CINCAE (Centro de investigación de la caña de azúcar del Ecuador) (Aguirre, 2015). 


\subsection{Características De La Energía}

(Mendoza J. , 2016) 1. Reacción; La energía se caracteriza por ser la causa inicial de toda reacción física o química, todo movimiento es producido por la energía, es la fuerza generadora de todo movimiento. (Torres, Salete, \& Flores, 2018) 2. Medición; La energía es medible; para cada energía existe un tipo de medición, ya sea térmica, química, o física de movimiento, incluso la energía nuclear y atómica tienen parámetros de medición. (Angulo, Martínez, \& López, 2017) 3. Almacenamiento: Muchas energías o fuentes de energía se pueden almacenar como los combustibles, el carbón y electricidad, lo que también permite su movilidad y traslado (Niño, 2018). 4. No se crea ni se destruye, Este principio consiste en que físicamente y químicamente la energía solo se transforma, no se puede crear ni se puede destruir, sólo se puede transformar. (Tarantino, 2018) 5. Degradación La energía se degrada, causa por la cual al haberse utilizado ya no puede aprovecharse nuevamente. (Videos, 2014) 6. Cambios: La energía tiene dos tipos de cambios físicos:

1. Cambios mecánicos. - Consisten en variaciones físicas de los objetos, como dilatación, deformación, elevación de temperatura etc.

2. Cambios térmicos. - Son los que producen una elevación o disminución de la temperatura.

\subsection{Tipos De Energías}

Según (Fernandez, 2018) Existen distintas clasificaciones posibles para la energía, según los aspectos en los que nos fijemos: eólica, solar, nuclear, calorífica, renovable, etc. Sin embargo, en Física se considera que existen 3 categorías fundamentales y la energía de un cuerpo o sistema puede pertenecer a uno solo o varios de las siguientes:

1. Energía interna: Debida a la composición y al estado del cuerpo (Navarro P. J., 2018).

2. Energía cinética: Debida al estado de movimiento del cuerpo (Muñoz, David, \& Santamaría, 2019).

3. Energía potencial: Debida a la posición que ocupa un cuerpo en un campo de fuerzas (Soto, 2020).

Por otro lado, podemos hacer una clasificación de tipo práctico, según el tipo de fuerzas implicadas o la forma de almacenamiento de la energía según su tipo de fuerza como se muestra en la tabla 1: 
TABLA 1. ALMACENAMIENTO DE ENERGÍA SEGÚN SU TIPO DE FUERZA

\begin{tabular}{|c|c|}
\hline Tipo de energía & Fuerzas implicadas o forma de almacenamiento \\
\hline \multirow{3}{*}{ Mecánica } & $\begin{array}{l}\text { Energía cinética: Asociada al movimiento de los cuerpos o sistemas (Navarro J. } \\
\text {,2018) }\end{array}$ \\
\hline & \\
\hline & $\begin{array}{l}\text { Energía potencial: Asociada a las fuerzas mecánicas: gravitatoria y elástica (Pe- } \\
\text { rez, 2018). }\end{array}$ \\
\hline \multirow{2}{*}{ Electromagnética } & $\begin{array}{l}\text { Energía de la corriente eléctrica y del campo electromagnético (Mendoza J., } \\
\text { 2019). }\end{array}$ \\
\hline & Asociada a las fuerzas eléctrica y magnética \\
\hline \multirow{4}{*}{ Luminosa o Radia } & Asociada al transporte de la radiación electromagnética (Anachuri, 2018) \\
\hline & \\
\hline & Energía de la luz visible y no visible (Intriago, 2018) \\
\hline & Asociada a la agitación interna molecular (Gómez, 2020) \\
\hline Térmica & Asociada al concepto de temperatura (Cuello, 2020) \\
\hline \multirow{4}{*}{ Química } & Energía de los enlaces químicos (Rufino, 2018) \\
\hline & \\
\hline & Aparece en las reacciones químicas (Garcia, 2018) \\
\hline & Energía de cohesión interna de los núcleos (Gallardo, Machado, \& Sanchís, 2018) \\
\hline Nuclear & Aparece en las reacciones nucleares (Live, 2019) \\
\hline
\end{tabular}

Fuente: Verdezoto, L (2021)

\subsection{Energía Renovable, No Renovable Y Limpia}

(Cardenas, Echeverria, \& Cepeda, 2015) 1) Energía no renovable. Es obtenida por vía del petróleo y del gas natural, los cuales por su naturaleza si se renovarían requerían de millones de años, una elevada presión y temperatura de la tierra. (Guachún, 2018) 2) Energía renovable. La energía renovable es la que es producida y al dejar sin usar se puede volver a producir desde cero; aquí podemos encontrar energías como son la solar, la eólica y la geotérmica. (Ancu, Bossa, \& Vives, 2018) 3) Energía limpia. Son todas aquellas energías que no causan daños al planeta. Entre las energías limpias podemos mencionar la geotérmica, la hidráulica, la eólica y la energía solar, biomasa, biogás y mareomotriz.

\section{METODOLOGÍA}

\subsection{Biomasa}

La biomasa es un tipo de energía renovable en la que se emplea materia orgánica. (Quintero \& Quintero, 2014) Esta materia orgánica es originada en un proceso biológico, espontáneo o provocado.

La energía de la biomasa proviene del sol. Mediante la fotosíntesis, el reino vegetal absorbe y almacena una parte de la energía solar que llega a la tierra. Las células vegetales utilizan la radiación solar para formar sustancias orgánicas a partir de 
sustancias simples y del $\mathrm{CO}_{2}$ presente en el aire (Cruz, 2016). El reino animal incorpora, transforma y modifica dicha energía. En este proceso de transformación de la materia orgánica se generan subproductos que no tienen valor para la cadena nutritiva o no sirven para la fabricación de productos de mercado, pero que pueden utilizarse como combustible en diferentes aprovechamientos energéticos, como es el caso del gasoil se muestra en la Figura 1 (Mejias, 2020).

Aproximadamente:

kilogramo de biomasa ( $0.15-0.30 € / \mathrm{kg}$ ) proporciona

400 kilocalorías, 1 litro de gasoil $(0.90-1 \in / 1)$ proporciona 8700 kilocalorías

Figura 1: Cantidad de biomasa proporcionada a kilocalorías Fuente: Benítez (2016)

\subsection{Transformación De La Biomasa En} Energía Renovable

La valoración de la biomasa puede hacerse a través de cuatro procesos básicos mediante los que puede transformarse en calor y electricidad: combustión, pirolisis, gasificación y digestión anaerobia (Tierradentro Cruz, 2020).

Combustión. Se somete a la biomasa a altas temperaturas con exceso de oxígeno. Es el método tradicional para la obtención de calor en entornos domésticos, para la producción de calor industrial o para la generación de energía eléctrica (Chong, 2018).

Pirolisis. Se somete a la biomasa altas temperaturas (alrededor de $500^{\circ} \mathrm{C}$ ) $\sin$ presencia de oxígeno. Se utiliza para producir carbón vegetal y también para obtener combustibles líquidos semejantes a los hidrocarburos (Pinedo, 2013).

Gasificación. Se somete a muy altas temperaturas en presencia de cantidades limitadas de oxígeno, las necesarias para conseguir así una combustión completa. Según se utilice aire u oxígeno puro, se obtienen dos productos distintos, en el primer caso se obtiene gasógeno o gas pobre (este gas puede utilizarse para obtener electricidad y vapor), en el segundo caso, se opera en un gasificador con oxígeno y vapor de agua y lo que se obtiene es gas de síntesis. La importancia del gas de síntesis radica en que puede ser transformado en combustible líquido (Romero, 2010).

Digestiónanaerobia.Sebasanenlautilización de diversos tipos de microorganismos que degradan las moléculas a compuestos más simples de alta densidad energéticas. La digestión anaerobia de la biomasa por las bacterias Acetobacter, se encargan de descomponer los ácidos grasos y compuestos aromáticos, en cuanto a las Eubakterium son capaces de cumplir funciones específicas en la descomposición anaeróbica. Se puede utilizar en explotaciones de ganadería intensiva, con la instalación de digestores o fermentadores, en donde la celulosa procedente de los excrementos animales se degrada en un gas que contiene cerca del $60 \%$ de metano (Gutierrez M. , 2014).

La combustión de biomasa no contribuye al aumento del efecto invernadero porque el carbono que se libera forma parte de la atmósfera actual (es el que absorben y liberan continuamente las plantas durante su crecimiento) y no del subsuelo, capturado en 
épocas remotas, precisamente como el gas o el petróleo (Martinez, 2012).

\subsection{Cogeneración}

Es un procedimiento mediante el cual se genera de forma simultánea energía eléctrica, mecánica y térmica (Lizárraga, 2020). El sector azucarero ha sido señalado por estudios nacionales e internacionales como el mayor potencial de cogeneración por su disponibilidad de biomasa, en especial el bagazo. Este subproducto, derivado de procesos de cosecha y molienda de caña, constituye la fuente primaria de energía para la cogeneración (Rodriguez, 2017).

En una central eléctrica tradicional los humos salen directamente por la chimenea mientras que, en una planta de cogeneración, los gases de escape se enfrían transmitiendo su energía a un circuito de agua caliente/vapor, una vez enfriados los gases de escape pasan a la chimenea, con el aprovechamiento del calor residual, los sistemas de cogeneración presentan rendimientos globales del orden del $85 \%$, lo que implica que el aprovechamiento simultáneo de electricidad y calor favorezca la obtención de elevados índices de ahorro energético, así como una disminución importante de la factura energética, sin alterar el proceso productivo. Ahorro energético que se incrementa notablemente si se utilizan energías residuales (Armengod, 2019). La producción energética basada en el petróleo y el carbón disminuye rápidamente y la energía nuclear decrece mientras que los ciclos de cogeneración se mantienen. Las tendencias energéticas apuntan a que las renovables son el único ámbito de la energía que crece y se desarrolla (Candal, 2021).

\subsection{Proceso de cogeneración}

Este proceso corresponde a la generación de energía como parte del proceso productivo, bien sea de azúcar o de etanol (Gonzales, Soto, Martinez, \& Zamora, 2017). Los ingenios desde sus inicios han utilizado el bagazo de la caña (combustible renovable) como combustible para alimentar sus calderas y utilizar el vapor como energía para el funcionamiento de sus procesos (Conteras, 2019). La cogeneración entonces utiliza la energía en forma de calor producida por el bagazo para generar vapor y luego mediante el uso de turbogeneradores, la energía eléctrica. El vapor de escape del turbogenerador entonces va al proceso productivo mientras que la electricidad es mayoritariamente utilizada para su consumo propio. Parte del bagazo también se utiliza en la industria papelera como fuente de fibra, lo cual evita la tala de árboles (Rodriguez, 2017).

\subsection{Ingenios Azucareros}

(Alderetes, 2016) Se estima que hay más de 1000 ingenios azucareros alrededor del mundo y teniendo en cuenta la cantidad de generadores de vapor instalados en cada uno de ellos, se puede decir que la industria azucarera sea entre las industrias, la de mayor demanda de calderas industriales. A nivel global puede estimarse entonces que existen más de 3000 calderas instaladas, solamente en los ingenios del Estado de San PabloBrasil (Cárdenas, 2018).

Por otro lado, el creciente interés y desarrollo de la cogeneración en la industria, trajo 
calderas de gran capacidad, eficiencia con elevadas presiones y temperaturas de trabajo (Amaya, 2014). Por otra parte, un replanteo integral de las prácticas operacionales y de mantenimiento, con calderas tradicionales de baja presión y deficiencia. Dependiendo del país y cogenerando a partir del bagazo, la industria azucarera aporta hasta más del 5\% de la energía total requerida por el mismo, generando importantes ahorros de combustibles fósiles no renovables (Chozo, 2019).

También sumándose a estos cambios, ya están en marcha en Brasil y otros países las primeras calderas de un solo domo (monodrum) y las de lecho fluidizado que introducen una nueva tecnología e innovación en este campo, con capacidades y condiciones de trabajo nunca antes visto, salvo en las centrales termoeléctricas (Velásquez, 2014).

El manejo e implementación de las prácticas están establecidas en los códigos y normas internacionales para calderas, tales como: la Sociedad Americana de Ingenieros Mecánicos (ASME), del National Board Inspector Code (NBIC), EPRI (Energy Power Research Institute) o las del Comité Europeo de Normalización (CEN), como también la adopción de nuevas técnicas en el campo del conocimiento que posibilitan un manejo más responsable y eficiente de este equipamiento. Nuevos conocimientos sobre las propiedades del bagazo, sumados a la aparición de herramientas computacionales de cálculo, diseño y simulación tales como CFD (Computational Fuid Dynamics), FEM (Finite Element Method), más un importante avance tecnológico en las áreas de la instrumentación, control automático, en técnicas no destructivas (NDT) de inspección y mantenimiento, etc. (Alderetes, 2016).

(Gozález \& Rodrigues, 2018) El máximo aprovechamiento de la biomasa cañera, con vista a la generación y comercialización de electricidad, resulta vital para la supervivencia de la industria azucarera moderna. (Quiróz, Coca, \& Baquero, 2016) El residuo en Colombia más importante derivado del procesamiento de la caña de azúcar es el bagazo que representa el 30\% de la caña molida, una porción aproximada de 15\%equivalente al (1089229 toneladas) es llevada a la generación de papel y el $85 \%$ restante es destinado a cogeneración de energía, como se muestra en la Figura 2. 


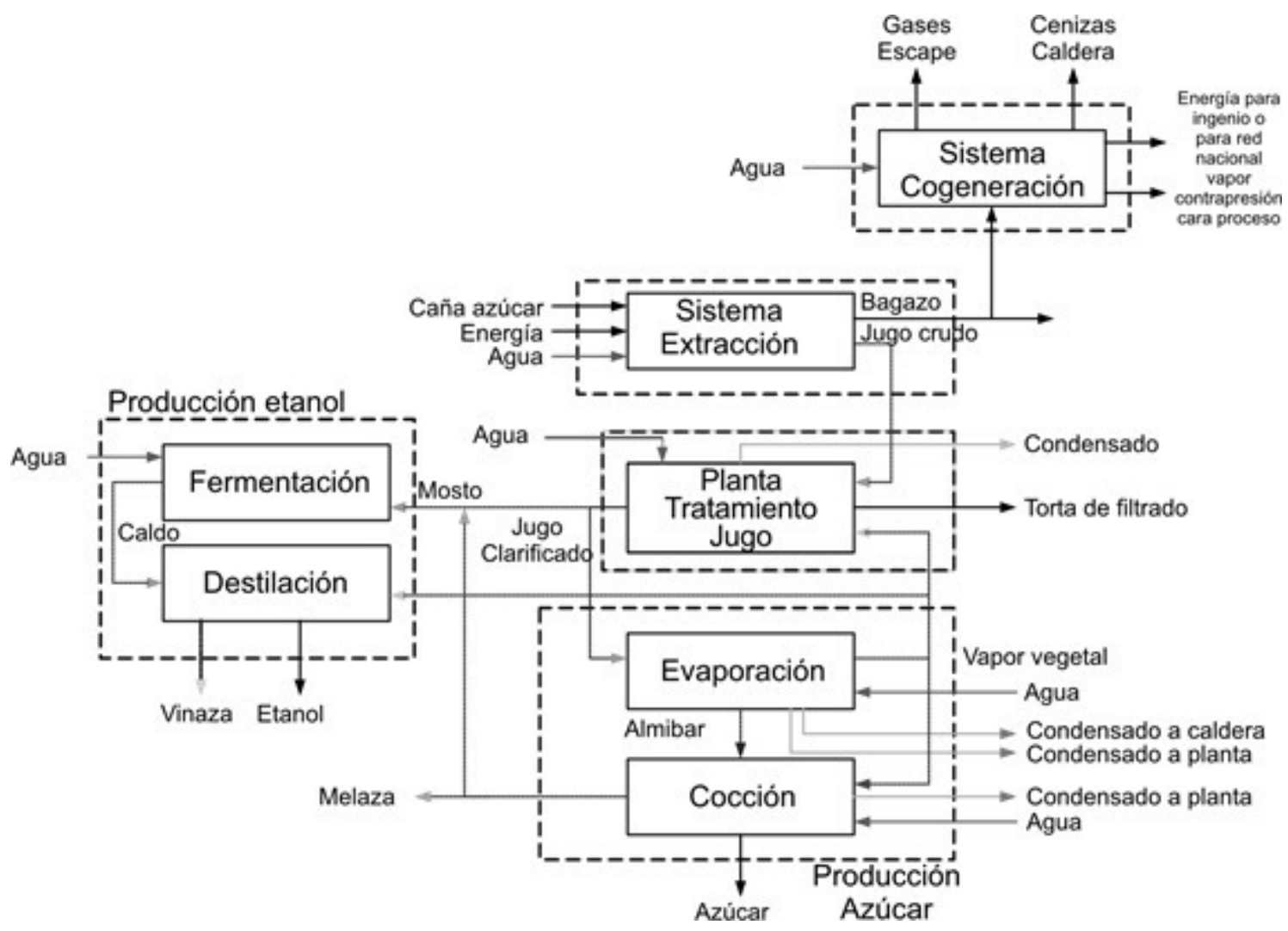

Figura 2: Producción de Etanol, Azúcar y Energía

Fuente: Oliveira y Pellegrini (2010)

\subsection{Industria Azucarera El Ingenio San}

\section{Carlos}

El Ingenio San Carlos es la industria azucarera más grande del Ecuador la cual está en la capacidad de producir anualmente alrededor de 2000,000 toneladas métricas de caña con lo que se genera una cifra cercana a los 3.050 .000 sacos de $50 \mathrm{Kg}$, de azúcar en distintas presentaciones. (Hoyos Lozada, 2019)Se produce también azúcar crudo para exportación $\mathrm{y}$, en menor medida para la satisfacción de necesidades del mercado nacional, se elaboran otros productos como azúcar impalpable, panela, panela granulada, miel de caña, etc. (Pinto Arboleda Andrés Rafael, 2015)

Además, a partir del año 2004, se inició la generación de excedentes de energía eléctrica en base a biomasa (bagazo de caña) que son vendidos al Mercado Eléctrico Mayorista (M.E.M.) del Ecuador, que los generan dos turbos generados de 12MW ,16 MW y a partir del año 2014 se incrementó un nuevo generador de 43 MW que trabaja en conjunto con una nueva caldera de 950 psig (Gutierrez A. , 2018).

\section{RESULTADOS Y DISCUSIÓN}

El bagazo obtenido en una etapa de producción, es una fuente renovable para la producción de energía calórica a través de la cogeneración, esto denota la importancia de la misma y el aprovechamiento como biocombustibles para las calderas de alta presión en los ingenios azucareros.

Algunas industrias, especialmente los ingenios azucareros ha sustituido los combustibles fósiles por bagazo de caña, en los últimos 
años los cogeneradores prácticamente han aumentado su consumo de bagazo, de esta manera se ha reducido el consumo de hidrocarburos por un combustible más barato y limpio. El bagazo de caña junto a sus propiedades permite que su combustión sea más eficiente, de esta manera se aprovecha la energía termina para la generación de vapor acetabulares. La generación De esta manera se perfila como una fuente de energía amigable con el medio ambiente, ya que las emisiones de dióxido de carbono son bajas.

La cogeneración indica el aprovechamiento máximo de ya sea en niveles de energía en una planta lo que trae consigo un aumento de los niveles de potencia térmica o eléctrica, con se obtiene un ahorro o maximización de costo de producción. De 48.9 ton/h de bagazo se genera 106 ton/h de vapor sobrecalentado

En los últimos años la utilización de biocombustibles como el bagazo de caña, ha tomado gran importancia en el ámbito académico, como industrial ya que las características que posee esta biomasa, hace que su combustión sea más eficiente y ecológica.

Según (Jaramillo, 2019) la Azucarera San Carlos en esta zafra del 2019 se obtuvo un total $28,412.00 \mathrm{Ha}$, con un rendimiento que fue de $183.00 \mathrm{lb}$. De Azúcar/T.M.C. su generación 211, 978, $174 \mathrm{KWH}$, con exportación de energía 211, 863, $760 \mathrm{KWH}$ en un sistema interno de 66, 699, $677 \mathrm{KWH}$.

Según (Salazar, 2018) el Ingenio Azucarero del Norte "IANCEM" produce 4600 hectáreas de caña de azúcar, la misma que dota de una producción total de 600.000 sacos de azúcar al año de $50 \mathrm{~kg} \mathrm{c} / \mathrm{u}$, llevando a cubrir los mercados de las provincias de Imbabura y Carchi, además genera un total de 189.35 de energía, teniendo un consumo propio de 3.756 .

Según (Quinteros, 2020) el ingenio azucarero Tababuela produce 330 toneladas de bagazo húmedo diariamente de las cuales el $90 \%$ es aprovechado en el sistema de cogeneración lo que significa que diariamente se usa 297 toneladas para que el sistema se mantenga operando normalmente, considerándose 10 días del año para los protocolos de mantenimiento.

Según (Gómez-Merino, 2017) el ingenio san Carlos produce 2 millones $300 \mathrm{mil}$ toneladas métricas de bagazo húmedo al año diariamente de las cuales se utiliza en un 30\% de cogeneración, en dos calderas de 40.909 $\mathrm{kg} / \mathrm{hr}$ de capacidad cada una, considerándose 3 meses al año se da mantenimiento.

\section{CONCLUSIONES}

El sistema de cogeneración bioenergética es una tecnología rentable por diversos motivos: se logra una independencia energética debida que se autoabastece, permitiendo eliminar considerables costos de consumo eléctrico. Teniendo un rendimiento eléctrico de $10 \%$ frente a las plantas termoeléctricas, por tanto, el poder calórico que aporta es aprovechable de mejor manera tiene una eficiencia del $13.3 \%$ comparado a la generación tradicional. Lo que caracteriza al proceso de cogeneración como eficiente es el porcentaje de perdida en la transmisión y distribución de energía eléctrica que es el $0 \%$ comparado con otros sistemas termoeléctricos. 
Con la ejecución del sistema de cogeneración en el ingenio azucarero San Carlos se ha confirmado la capacidad de generación térmica y eléctrica que esta puede tener, además de poder determinar todo el potencial que posee una hectárea de caña de azúcar referente a los sistemas de cogeneración, el sistema será más eficiente siempre y cuando se invierta en tecnología y el sistema de extracción de jugo de en la caña de azúcar sea el más óptimo para tener un bagazo con menor humedad y así aumentar el poder calorífico del mismo.

El uso de sistemas de cogeneración con biomasa permite aminorar la contaminación provocada por los gases de efecto invernadero producidos en las calderas de los ingenios azucareros dejando de quemar combustibles fósiles, que esta fábrica posee de sus procesos industriales en la elaboración de azúcar.

\section{REFERENCIAS BIBLIOGRÁFICAS}

Abreu, E., González, C., Rico, R., Morales, Z., \& Espinosa, P. (2016). Evaluacion de esquemas de cogeneracion de energia a partir de bagazo de caña de azucar. Centro Azucar, 89-92.

Aguilar, D. (2019). Hoy en día muchos estudios salen a flote con respecto a la biomasa para la generación de energía. Cabe recalcar que lo residuos sólidos urbano pueden ser aprovechados en para la cogeneración de energía. Según (Bohórquez, 2019) El potencial energético de l. Obtenido de Univsidad Politecnica Saleciana de Cuenca : https://dspace.ups. edu.ec/bitstream/123456789/18090/1/

\section{UPS-CT008600.pdf}

Aguirre, J. (2015). Estudio del Sistema de Cogeneracion a partir de la biomasa en los Ingenieros Azucareros. Revista Universidad Catolica de Santiago de Guayaquil, 5-7.

Alderetes, C. (2016). Calderas a Bagazo. Universidad de Belgrano Argentina, primera edicion.

Alexis Alonso-Gómez, A. B.-P. (2018). Materias primas usadas para la producción de Biomasa . Agrociencia , 12-23.

Amaya, D. (2014). Analisis y optimizacion exergetica de una planta de cogeneracion para la industria azucarera. Colombia: Universidad tecnologica de Pereira.

Anachuri, A. (2018). Determinacion y analisis de los niveles de contaminacion de electrosmog (densidad de potencial electromagnetica).

Aregentina:

Universidad Nacional del Comahue.

Ancu, S. P., Bossa, C. D., \& Vives, B. A. (2018). Energías Limpias Alternativas para el Desarrollo Organizacional. Santa Marta .

Angulo, A., Martínez, F., \& López, G. (2017). Almacenamiento de energía usando ultracondensadores ensistemas fotovoltaicos autónomos. Revista Visión Electrónica, 30-39.

Armengod, D. (2019). Cogeneración: energía eficiente para la industria. Digital Energy. Obtenido de Fao: https://www. energetica21.com/articulos-y-entrevistasonline-ver/cogeneracion-energiaeficiente-para-la-industria 
Aroca, D. (2015). Cogeneración: Qué es y cómo funciona. Ventajas y desventajas. Obtenido de Manufactoring: https:// leanmanufacturing 10.com/cogeneracionque-es-y-como-funciona

Asocaña. (2017). Más que azúcar, una fuente de energía renovable para el país. Sector agroindustrial de la caña, 1-3.

Benitez, L. (2016). Biomasa Canera. Ecured, $35-41$.

Blanco, N., \& Arce, E. (2014). Evaluacion Economica y Social del uso de la cana de azucar. Tecnologia en Marcha, 3-9.

Bohórquez, N. (2019). Generación de Energía a partir de Residuos Sólidos Urbanos. Estrategias Termodinámicas para Optimizar el Desempeño de Centrales Térmicas. Scielo, 2. doi:http://dx.doi. org/10.4067/S0718-07642019000100273

Candal, B. (2021). La nueva energía. Digital Energy, 1-2. Obtenido de https://www. energetica21.com/noticia/smart-energyla-nueva-energia

Cárdenas, K. (2018). Manejo adecuado de las emisiones atmosfericas en la industria del reciclaje de baterias de plomo. Bogotá: Universidad distrital francisco Jose de Caldas.

Cardenas, V., Echeverria, D., \& Cepeda, J. (2015). Energia renovable no convencionales en los sistemas de potencia. Revista Tecnica “Energia”.

Castro, B. (2017). Generación de energía a partir de bagazo de caña de azúcar para el Ingenio Zucarsa S.A. Guayaquil Ecuador: Universidad Espíritu Santo.
Chiriboga, A. (2018). Estudio técnico de un sistema para producir energía eléctrica a partir del biogas generado por la descomposición de la basura en la ciudad de Guayaquil. Repositorio institucional de la Universidad de Guayaquil, 22-36.

Chong, H. (2018). Análisis para la obtención de energía eléctrica utilizando un Gasificador de Biomasa. GuayaquilEcuador: Universidad Católica de Santiago de Guayaquil.

Chozo, L. (2019). Control de calidad en el proceso de cogeneración de energía en la Empresa Agrolmos S.A. distrito de Olmos, región Lambayeque. Peru: Universidad Nacional Pedro Ruiz Gallo.

Claudio Carpio, M. F. (2013). Eficiencia energética en América Latina y el Caribe: avances y desafíos del último quinquenio. CEPAL, 12-23.

Conteras, J. (2019). Simulación de una caldera bagacera mediante el software Aspen Plus para determinar su desempeño energético. Colombia: Universidad Autónoma de Bucaramanga.

Corrales, L. A. (2017). PLANESTRATEGICO DELAEMPRESARIOPAILAENERGIAEN EL MUNICIPIO DE ZARZAL, VIGENCIA 2017-2020. Obtenido de Univalle: https://bibliotecadigital.univalle.edu. co/bitstream/handle/10893/14995/CB0577766.pdf? sequence $=1 \&$ isAllowed $=\mathrm{y}$

Cruz, C. (2016). Oportunidades y barreras para el desarrollo de las energías renovables en Costa Rica. Funcion Cientec, 2-4.

Cuello, A. (2020). Determinación de 
propiedades térmicas para la congelación de la pulpa de carambola. Puerto Maldonado: Universidad Nacional Amazonica.

Debernardi De La Vequia; Laurel Ortiz; Calleja Rosas. (2016). Energia disponible a partir de la biomasa de la cana de azucar. Agroproductividad, Vol. 9, P. 68-73.

Debernardi-De La Vequia, H. e. (2016). "ENERGIA DISPONIBLE A PARTIR DE BIOMASA DE RESIDUOS DE CAÑA DE AZUCAR (Saccharum spp.)." . AGROProductividad, P 1-6.

Debernardi-De La Vequia, H.-L. H., \& Rosas-Calleja, D. (2016). energía disponible a partir de biomasa de residuos de caña de azúcar (saccharum spp. Agro Productividad, 68-73.

Espinosa-Mancinas, C. A. (2017). Papel artesanal de paja de azúcar. Agro productividad, 2-97.

Fábio Ricardo Marín, M. A. (2018). Modelación de la caña de azúcar en latinoamérica,Asdrúbal Farías,Fernando Villegas. Euroclima , 7- 24.

Fernandez, J. (2018). Energia, caracteristica y tipos. Scielo.

Gallardo, C., Machado, C., \& Sanchís, J. \&. (2018). Cosmología y conceptos fundamentales de la física contemporánea. Digibug, 8-12.

Garcia, Z. (2018). Enlace químico, orbital molecular y ontología. Dialnet, 93-109.

Gómez, M. (2020). Reducción de las pérdidas de flujo de calor mediante el uso de aislantes térmicos. Departamento de Tecnología de Alimentos, 4-6.

Gómez-Merino. (2017). $R$ esiduos organicos de la agroindustria. Mexixo.

Gonzales, C., Soto, L., Martinez, Y., \& Zamora, M. (2017). Análisis de cogeneración para satisfacer las demandas de las producciones integradas de azúcar y alcohol. Economia Quimica, 4-12.

Gozález, A., \& Rodrigues, M. (2018). Integración y esquemas energéticos para el máximo aprovechamiento de la biomasa cañera en la generación de electricidad. Scielo. Obtenido de http:// scielo.sld.cu/scielo.php? script $=$ sci xt\&pid=S2223-48612018000400003

Guachún, V. L. (2018). “Estudio de factibilidad de un sistema de energía renovable aplicado a un nodo de servicio de telecomunicaciones". CuencaEcuador .

Gutierrez, A. (2018). Industria Azucarera. EL COMERCIO, 23-28.

Gutierrez, M. (2014). Co-Digestion anaerobia de lodo con edar con residuos organicos con diferente naturaleza: combinacion de tecnicas experimentales y herramientas matematicas. Dadun, 14-22.

Hernandez, A., Gonzalez, V., \& Freide, M. (2017). Fuente de Biomasa para entregar electricidad. Centro Azucar, 1-8.

Hoyos Lozada, J. C. (2019). “La integración vertical de la cadena productiva del azúcar refinado. concentración de mercado, los precios y la inflación. Ambato -Ecuador. 
Intriago, D. (2018). Sistemas VLC Para Comunicaciones e Iluminación Indoor. Grupo de Sistemas Ópticos, 22-36.

Jaramillo, I. C. (2019). Sociedad Agricola e Industrial San Carlos S.A. Cana Verde, $2-12$.

José Serrano, W. M. (2017). Determinación del Potencial de Generación Eléctrica a partir de biomasa . Revista de la Facultad de Ciencias Químicas, 1-21.

José Serrano, W. M. (2017). Determinación del Potencial de Generación Eléctrica a partir de biomasa ecuador. Revista de la Facultad de Ciencias Químicas, 7-21.

Largo, M. (2015). Mejoramiento de la rentabilidad con diversificación de sub-productos de la caña azúcar, en chaguarpamba. loja. Loja: Lr15a.

Live, T. (2019). Política exterior del Ecuador frente a la agenda internacional de Energía Atómica (2007 - 2014). Quito: Quito, Ecuador : IAEN.

Lizárraga, R. (2020). Cogeneración de Energía. Mexico: Universidad tecnologica de altamira.

Marroquin, M. (2015). Análisis técnico económico del incremento de la cogeneración mediante la optimización del uso de vapor con biomasa cañera y la instalación de un turbogenerador en un ingenio azucarero. USAC, 12-22.

Martinez, R. (2012). Ingenieria basica de una planta de cogeneracion con biomasa. España: Universidad de Cantabria.

Mejias, W. (2020). Los principios basicos de la materia y la energia. Revista Universidad Internacional de Valencia, Vol. 6, 60-66.

Mendoza, J. (2016). Caracterisiticas de la energia. Dialnet.

Mendoza, J. (2019). Sistema electrónico portátil para la recarga eléctrica de dispositivos móviles mediante la captación de energía electromagnética. Ambato - Ecuador: Repositorio Universidad Técnica de Ambato .

Muñoz, M. Y., David, A. R., \& Santamaría, S. J. (2019). Evaluación del potencial de energía solar en Santander, Colombia. Prospectiva, 7-12.

Navarro, J.(2018). Energía potencialyenergía cinética. Ejemplos de Transformación de la energía potencial en energía cinética y viceversa. Lima, Perú: MONOGRAFÍA TRABAJO, POTENCIA Y ENERGÍA.

Navarro, P. J. (2018). Potencia y energía. Lima, Perú.

Niño, A. B. (2018). Microturbina pelton, una solucion real de energia para zonas no interconectadas (zni). Revista Colombiana de Tecnologías de Avanzada, 72-76.

Oliveira, S. D., \& Pellegrini, L. (2010). Plantas de Cogeneracion de la Cana de Azucar. Palmas, 30-31.

Pantaleon. (12 de 04 de 2017). Experiencias en cogeneración. Obtenido de Pantaleon: http://www. quitecsa.com/phocadownload/ Cogeneraci \%c 3\%b3 n \% 20 en $\% 20$ Ingenios\%20Azucareros.pdf

Paz-Enrique, L. E., Hernández-Alfonso, 
E. A., \& Peralta-González. (2016). Productividad científica de la temática caña de azúcar en la Web of Science. ICIDCA, 44-49.

Perez, J. (2018). Potencia. Eficiencia mecánica. Lima, Perú : Monografia trabajo, potenccial y energia.

Pinedo, A. (2013). Obtencion de bicarbonato $y$ combustibles mediante pirolisis de biomasa residual. Peru: Consejo Superior de investigaciones cientificas.

Pinto Arboleda Andrés Rafael, V. R. (2015). Producción de un video institucional del ingenio san carlos. Guayaquil-Ecuador.

Pozo, R. V., \& Suárez, A. M. (2018). Comportamiento agronómico de ocho variedades de saccharum officinarum l., caña soca años 1 y 2, en río verde, provincia de santa elena. La Libertad.

Quintero, G. J., \& Quintero, G. E. (2014). Energía obtenida a partir de biomasa. Investigacion, Innovacion, Ingenieria, 28-44.

Quinteros, O. (2020). Caracterizacion del bagazo de caña de azucar de variedades del Ingenio Azucarero Tababuela para Bioenergia. Ibarra-Ecuador: Universidad Tecnica del Norte.

Quiróz, A. P., Coca, A., \& Baquero, P. (2016). Sostenibilidad del Aprovechamiento del Bagazo de Caña de Azucar en el Valle de Cauca, Colombia. Researchgate, 135.

Rodríguez, D. C., \& Fernández, X. S. (2014). La Producción De Energía Eléctrica a Partir de la Biomasa Forestal Primaria: Análisis del Caso Gallego. Revista Galega de Economía, 91-111.

Rodriguez, M. (2017). Mas que azucar una fuente de energia renovable. ASOCANA, 21-30.

Romero, A. (2010). Aprovechamiento de la biomasa como fuente de energia alternativa a los combustibles fosiles. R.Acad.Cienc.Exact.Fís.Nat. (Esp), 4-8.

Rosero, Garcia, Minchala, Pozo, \& Morales. (2013). Fuentes de Generación de Energia Eléctrica Convencional y Renovable a Nivel Mundial. Revista Politénica, 1. Obtenido de https://revistapolitecnica. epn.edu.ec/ojs2/index.php/revista_ politecnica2/article/download/217/ $\operatorname{pdf} / 5755$

Rosero, J., \& L. Garza, L. M. (2016). Fuentes de Generación de Energía Eléctrica Convencional y Renovable a Nivel Mundial. Renovable Sources, 1-13.

Rufino, L. (2018). PROGRAMACIÓN ANUAL DE FÍSICA Y QUÍMICA PARA $2^{\circ}$ DE EDUCACIÓN SECUNDARIA OBLIGATORIA Y SITUACIÓN DE APRENDIZAJE ENERGÍA TÉRMICA $Y$ TEMPERATURA. Santa Cruz: Universidad de la Laguna.

Salazar, V. J. (2018). Estrategias de control interno para el Ingenio Azucarero del Norte "IANCEM" cem. Ibarra - Ecuador.

Sardiñas,

A.

$\mathrm{H}$. (2017). APROVECHAMIENTO DE las posibles fuentes de biomasa para entregar más electricidad en la fábrica de azúcar antonio sánchez. Centro Azúcar, 88-97. 
Energía Cinética y Potencia. Revista Vida

Cientifica , 43-44.

Sousa, L. N. (2018). Potencial de la Cana de Azucar como fuente de energia para America Latina y el Caribe. Educacion Ambiental, 50-62.

Tarantino, S. C. (2018). Propuesta teórica: degradación de energía en los niveles jerárquicos de toda organizacion. Revista Ingenieria de Negocios .

Tierradentro Cruz, J. \&. (2020). Aprovechamiento de la biomasa residual pecuaria en Colombia. Mare Ingenii. Ingenierías, 1(2), 46-55.

Tigua, E. (2017). Modelo de mejora continua para el proceso de molienda en el ingenio azucarero Monterrey Malca. CuencaEcuador.

Torres, Salete, W. M., \& Flores, C. C. (2018). Metodología para el seguimiento, medición y análisis energético de una planta manufacturera. Revista Universidad y Sociedad, 78-84.

Valdes, A., Lopez, P., \& Isaac, J. (2017). Energia Renovable a partir de la Biomasa de la Cana de Azucar. Revista Ciencia, Tecnologia y Medio Ambiente, 11-16.

Velásquez, A. (2014). Análisis de Calderas para Generación de Energía Eléctrica. Guayaquil, Ecuador: Universidad Catolica de Santiago de Guayaquil.

Videos, R. (2014). Auscultación Hidroeléctrica Piedra del Águila Evaluación de Alternativas de cambio del Sistema Telemedido de Auscultación (STA). Córdoba : CRUC-IUA UNDEF.
Obtenido de Pantaleon: http://www. quitecsa.com/phocadownload/ Cogeneraci\%c3\%b3n\%20en\%20 Ingenios\%20Azucareros.pdf

Zumalacagui, L. (2015). Potencialidades del bagazo para la obtencion de etanol frente a la generacion de electricidad. Ingenieria, Investigacion y Tecnologia, 11-16. 